\title{
Safety Requirements for the Design of Collaborative Robotic Workstations in Europe - a review
}

\author{
Carlos Faria ${ }^{1, *}$, Ana Colim ${ }^{1}$, João Cunha ${ }^{1}$, João Oliveira ${ }^{1}$, \\ Nelson $\mathrm{Costa}^{2}$, Paula Carneiro ${ }^{2}$, Sérgio Monteiro ${ }^{2}$, Estela Bicho ${ }^{2}$, \\ Luís A. Rocha ${ }^{1}$ and Pedro Arezes ${ }^{2}$ \\ ${ }^{1}$ DTx-Colab, Guimarães, Portugal \\ \{carlos.faria ${ }^{*}$, ana.colim, joao.cunha, joao.oliveira, luis.rocha\}@dtx-colab.pt \\ ${ }^{2}$ Centre Algoritmi - Universidade do Minho, Guimarães, Portugal \\ \{ncosta, pcarneiro, parezes\}@dps.uminho.pt \\ \{sergio, estela.bicho\}@dei.uminho.pt
}

\begin{abstract}
Industrial manufacturing is moving towards flexible and intelligent processes. Human-Robot Collaboration (HRC) has a pivotal role in smart factories due to a more versatile resource allocation that ultimately drives higher productivity and efficiency. The physical barriers that separate robots' and humans' workspaces are removed to facilitate HRC, which raises new safety concerns. To cope with this new robotics paradigm, regulatory legislation and international safety standards have been issued and are enforced for any machinery placed in factories. In this paper, we aim to shorten the gap between research projects and industry-ready robotic systems, by providing the guidelines and general requirements for collaborative robotic applications. We review the current international safety standards, certification procedures under the scope of European jurisdiction, and elaborate a literature review of papers related to safety for collaborative workstations.
\end{abstract}

Keywords: Industrial Safety · Collaborative Robotics · International Standards

\section{Introduction}

Industrial robots are an integral part of modern manufacturing processes. Robots are programmable agents capable of repetitively and consistently performing tasks with a high degree of precision. They are also applied to tasks deemed unhealthy or too dangerous for humans [1]. At the same time, robots are powerful machines, capable of generating large forces and torques with unanticipated or unpredictable movements for the operator; a prospect that poses a serious and immediate risk to the operator. The safety of the human operator is generally guaranteed by the physical separation of the operator's and the robot's workspaces [2-4].

Even though industrial robots are a staple in today's industry, there are some tasks that cannot be easily robotized due to their complexity or variability. To overcome this limitation, roboticists explored the idea of closing the distance between robot and operator in order to create a collaborative space, where the robot's consistency and precision are complemented by the operator's flexibility and fast decision making. This idea 
crystallized into a concept known as collaborative robotics a branch of robotics where the human-robot interaction is permissible and often desirable.

This paradigm shift means that barriers which once separated the workspaces of operators and robots are now removed to facilitate human-robot collaboration (HRC). The human/robot proximity and hybrid task assignment raise questions about the safety of the operators and of their surroundings. Moreover, the shared, unstructured and dynamic environments only add to the complexity of the risk assessment process.

Regulatory agencies such as the International Organization for Standardization (ISO), the Canadian Standards Association (CSA) or the American National Standards Institute (ANSI), have produced standards directed to the design and development of robot systems, their integration, and more recently, standards that specifically target collaborative robots - cobots. These serve as basic guidelines to identify possible hazards, evaluate them and reduce the risk of accidents.

In this document, we sought to address the key documentation and findings regarding safety for collaborative robotic workstations under the scope of European jurisdiction. The legal documents and International Standards applicable in this domain were also revised.

\section{Legal Base}

The first step into the certification process passes through the regulatory terms incited by the European Commission (EC). In Europe, machinery is required to comply with EC directives in order to be eligible for commercialization or usage within European borders. Robot systems available in Europe should be ratified against the Machinery Directive (2006/42/EC) [5] and the Use of Work Equipment Directive $(2009 / 104 / E C)$. These directives are upheld in each member state by their own set of law decrees. The directives define a set of legally binding obligations for manufacturers and sellers that favors the integration of safety parameters during the project phase.

The manufacturer or integrator of the robot system shall conduct a risk assessment procedure and meet the relevant essential health and safety requirements from the Annex I of the Machine Directive. Moreover, the technical files (indicated in Annex VII, part A), as well as any other necessary information, shall be provided. Once appropriate procedures to assess conformity are carried out, the EC declaration of conformity can be drawn up and the CE marking consequently affixed.

If the robot application is not listed in Annex IV - categories of machines capable of inducing severe injuries - the system integrator shall apply the procedure for assessment of conformity with internal checks on the manufacture of machinery (Annex VIII). Instead, if the robot system is listed in Annex IV, the integrator shall conduct one of the following procedures:

1. Internal checks on manufacture (Annex VIII);

2. EC type-examination (Annex IX) combined with the internal checks on manufacture (Annex VIII, point 3);

3. Full quality assurance procedure (Annex X). 


\section{Safety Standards}

International Standards play a key role in setting the baseline to what constitutes best practices in safe system behavior, and in design methodology. Although compliance with standards is not mandatory, they are considered as valid regulatory guidance when recognized by the European Committee for Standardization (ECS). Standards capture the international consensus and set general guidelines to certify the "mission-worthiness" of new products/systems, which also accelerates their commissioning process.

The ECS cooperates with international bodies such as the International Organization for Standardization (ISO) and the International Electrotechnical Commission (IEC) to produce harmonized European norms. In the scope of Collaborative Robotics, the relevant international standards are summarized in Fig. 1.

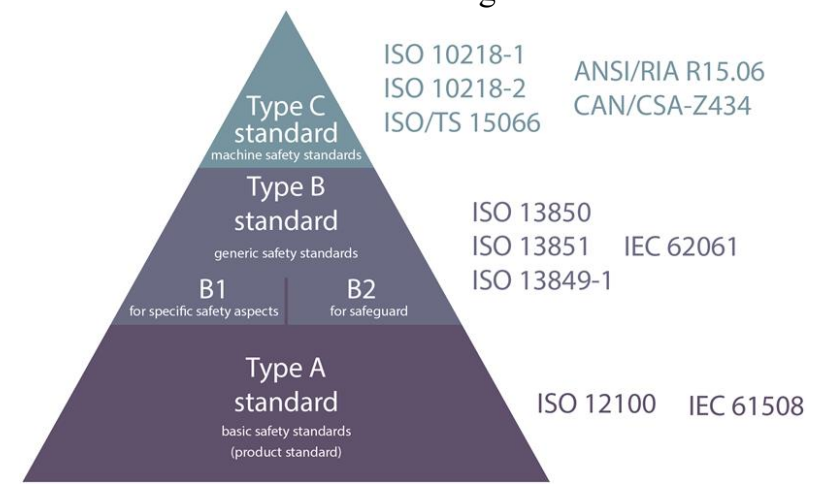

Fig. 1. Categories of Safety Standards according to International Standards Organizations. Other associations such as the American National Standards Institute (ANSI) and the CSA Group (formerly the Canadian Standards Association) exist and have published norms related to Collaborative Robotics. Due to regulatory differences between the EU/EEA and the U.S., the EC tends to work with organizations that produce globally relevant standards, for example, ISO and IEC.

IEC/ISO machine safety standards are organized in a three-level hierarchy from the most general and relatable to generic machinery - type $A$ - to machine-specific - type $C$. Type $C$ norms should be used as templates and take precedence over standards that are more general. In any case, different standard types are not dissociated, as type $C$ norms often refer to standards of types A and B to address, for example, risk assessment and other safety-related details.

Type A norms establish basic concepts, conception principles and general requirements applicable to machinery. These define the strategy for risk assessment and risk reduction. The risk assessment involves the i) determination of the robotic system limits, ii) identification of risks and hazards that may occur during standard operation, commissioning, or maintenance, iii) risk estimation, and iv) risk evaluation. In the risk assessment process, the evaluator may combine different tools to determine the risk level based on damage severity, frequency or time of exposure, and the possibility to limit or avoid damage.

Type $B$ norms specify safety aspects or safeguards applicable to machinery. These norms are split into two categories, the $B 1$ norms related to general safety aspects (e.g., 
safety distances, surface temperature, noise levels, etc.), and the $B 2$ norms about safeguard (e.g., bimanual commands, interlock devices, pressure-sensitive devices, protective equipment, etc.). Type $B$ norms describe mechanisms to identify the safety functions, to determine the category ${ }^{1}$ and required performance levels (PLr) for safety-related parts of the control systems (SRP/CS). The required performance level is determined after the risk level previously determined during risk assessment (ISO 12100). It is the robotic system integrator's responsibility to guarantee that the robot and any other parts of the system are accompanied by a certificate of conformity that attests to the part's performance level (PL) and category, and that it meets the expected PLr.

Type $C$ norms identify specific safety requirements applicable to a machine category. The norm ISO 10218 divides into part 1 - provides guidance for the design and construction of robots, and part 2 - that focuses on the integration process of robotic systems. Integrators of cobot workstations that operate with commercial robots should focus on part 2, which defines guidelines for safeguarding personnel, commissioning, functional testing, programming, operation, and maintenance.

The norm ISO 10218-2 narrows down the scope of the risk assessment process, by focusing on topics relatable to robotic systems. First, a list of robot limits is proposed and organized by topic: usage, space, time, and others. Then, ISO 10218-2, Annex A, compiles a list of the most significant hazards of different sources (mechanical, electrical, thermal, noise, etc.) relatable to robotic applications. The norm proceeds to address the safety requirements and protective measures. It extends and particularizes requirements for SRP/CS in the aforementioned type $B$ norms to the address robot applications. The layout design contemplated in this norm rests primarily in the use of physical or electro-sensitive equipment to delimit the robot and operator's workspace. Finally, the Annex G of the ISO 10218-2 includes a comprehensive table to verify the compliance of the safety requirements and measures for robotic systems.

The topic of collaborative robotics is referred to in the ISO 10218-2 (section 5.11 and Annex G), but given the complexity of the subject, it is supplemented by the technical specification ISO/TS 15066. In collaborative applications, the interaction between humans and robots is permitted and often desired. To facilitate this interaction, the physical limits that safeguard the robot's workspace are often removed. With a shared workspace, the safety of the operator is guaranteed by inherently safe design measures or safety-rated limiting functions.

The foreseeable human-robot interaction is categorized by the ISO/TS 15066 in four collaborative operation modes as depicted in Fig. 2.

The SMS is a black and white approach to collaborative robotics applications. The operator and robot may co-exist in the same space, although they shall not operate within the collaborative workspace at the same time. If the safeguard perimeter is breached, a safety-rated monitored stop ceases the robot motion.

The HG mode is an extension to the SMS collaborative mode, it functions based on the same premise, only this time, the system also comprehends a hand-guidance device to directly maneuver the robot when the operator is within the safeguard perimeter.

The SSM mode permits the robot and the operator to move concurrently within the collaborative workspace. The risk reduction is achieved by maintaining the protective

\footnotetext{
${ }^{1}$ The control architecture typologies (number and relationship between input, logic, test, and output elements) are labeled in different categories (B, 1, 2, 3, 4).
} 
separation distance at all times. A tracking device continuously monitors the distances between the hazardous robotic system parts and any person within the collaborative space. The robot operating speed is proportional to the shortest calculated distance.

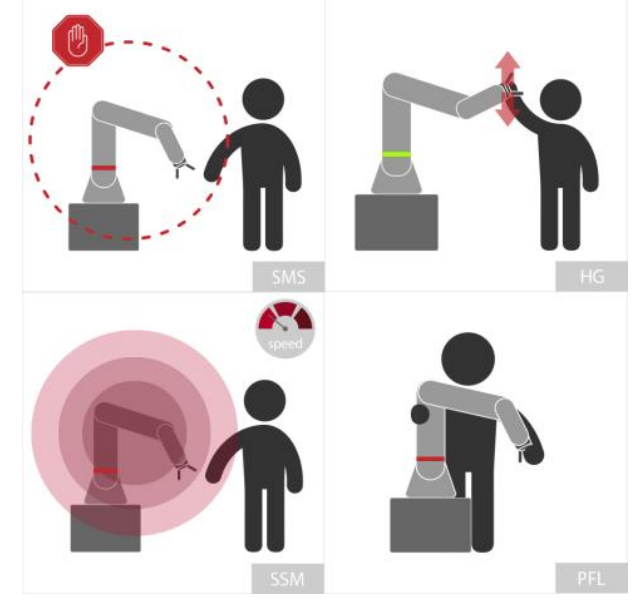

Fig. 2. Collaborative operation modes, Safety-rated Monitored Stop (SMS), Hand-Guiding (HG), Speed and Separation Monitoring (SSM), and Power and Force Limiting (PFL).

The PFL mode is the only collaborative mode that permits contact between the robot and the operator. Instead of separation, the risk reduction is achieved through inherently safe means or safety-rated control systems that keep hazards below a threshold. These include passive measures in the robot design (larger contact surface area through edge smoothing, padding or deformable parts, and the limitation of moving masses); and active measures in the robot control system (torque/force limiting, use of safety-rated soft axis/space limits, safety-rated monitored stop functions).

\section{$4 \quad$ Literature review}

To extend the revision of the legal and normative requirements, we conducted a literature review focused on safety requirements for cobot workstations using the keywords safety, collaborative robotics, HRC, and international standards; in four databases: Scopus, PubMed, IEEEXplore, and ScienceDirect. Papers from the last 5 years were revised and grouped into state-of-the-art reviews, innovative safety solutions, risk assessment and analysis methods.

Extensive state-of-the-art reviews on safety standards and measures can be found in [1], [6] and [7]. Robla-Gomez et al. analyzed current legal regulations and the main safety systems applied in industrial robotics that contribute to safe HRC [1]. Finkemeyer discussed the potential benefits and possible use cases of HRC, safety functions, standards, and technical issues [6]. Murashov et al. summarized safety standards and recommended measures that guarantee the safety of human workers at all times [7].

Different innovative safety solutions were proposed, and experimentally validated, in [8], [9], [10], [11], and [12]. Navarro et al. presented an adaptive damping controller 
that fulfills ISO 10218 and enables the operator to manipulate the robot safely without the risk of exceeding the velocity, power and force constraints [8]. The controller was validated in an industrial screwing application. Rojas et al. developed a trajectory planning method based on safe minimum-jerk trajectories that ensure physical safety and reduces psychological strain [9]. As a proof-of-concept, the authors presented an experimental setup and provide insights on the implementation of the proposed method. Vysocky et al. proposed a motion planning strategy that considers safety precautions stipulated in ISO/TS 15066 [10]. The strategy was tested in an experimental workstation with the assistant co-working manipulator $\mathrm{PaDY}$. The results show a reduction of velocity and of the risk of harming workers during robot operation. Zanchettin et al. developed a kinematic control strategy that enforces safety while maintaining productivity [12]. The methodology was experimentally validated on a dual-arm concept robot performing a manipulation task. Lasota and Shah conducted an experiment in which participants performed a collaborative task with an adaptive robot that incorporates human-aware motion planning and with a baseline robot using shortest-path trajectories [11]. Results show that working with the adaptive robot allowed participants to complete the task faster, with more concurrent motion, with less human and robot idle time, and with a larger human-robot separation distance. Participants also indicated they were more satisfied with the adaptive robot as a teammate and felt safer and more comfortable working with it.

Methodologies and guidelines for risk assessment are provided in [4], [13], and [14]. Vicentini et al. proposed a risk analysis methodology for HRC applications compatible with ISO standards that relied on a formal verification technique to automate traditional risk analysis methods [13]. Rosenstrauch and Kruger introduced safety standards and guidelines for risk assessment in general and industrial robots specifically, followed by a detailed insight of ISO/TS 15066 [4]. Poot, Johansen, and Gopinath presented a thorough risk assessment for an automated warehouse, where mobile robots and humans collaborate in a shared workspace [14]. Risk assessment is performed using a Hazard and Operability Study (HAZOP) to identify hazards, coupled with Unified Modeling Language (UML) and risk estimation. In this work, one can find a systemic description of collaborative scenarios and interactions, human roles, and unsafe scenarios.

\section{Conclusions}

HRC is an appealing prospect to the industry in general: to small and medium-sized enterprises (SME) due to the high degree of adaptability and flexibility, as well as to mass production companies that are rapidly shifting into mass customization. Moreover, the interaction between humans and robots is growing wider - with an increasing range of products fabricated with the assistance of precise and repeatable robotic platforms - and deeper as the human-machine interaction moves beyond the physical cages. The quest for a safer human-robot workstation grew considerably more complex with the lack of physical separation between the human and the robot workspaces. Labeling a product or system as safe is a substantial claim, one that is necessarily supported by results and data, and confirmed by satisfactory compliance assessment. We reviewed the key documentation related to the design of collaborative robotic applications, the 
legislation enforced in Europe, the harmonized international standards, and the latest publications on the field.

\section{Acknowledgments}

This work was supported by NORTE-06-3559-FSE-000018, integrated into the invitation NORTE-59-2018-41, aiming to hire highly-qualified human resources, co-financed by the Regional Operational Programme of the North 2020, thematic area of Competitiveness and Employment, through the European Social Fund (ESF).

\section{References}

1. Robla-Gomez, S., Becerra, V.M., Llata, J.R., Gonzalez-Sarabia, E., Torre-Ferrero, C., Perez-Oria, J.: Working Together: A Review on Safe Human-Robot Collaboration in Industrial Environments. IEEE Access. 5, 26754-26773 (2017).

2. International Organization for Standardization: ISO 10218 Robots and robotic devices Safety requirements for industrial robots - Part 2: Robot systems and integration, (2011).

3. International Organization for Standardization: ISO/TS 15066 Robots and robotic devices - Collaborative robots, (2016).

4. Rosenstrauch, M.J., Kruger, J.: Safe human-robot-collaboration-introduction and experiment using ISO/TS 15066. 2017 3rd Int. Conf. Control. Autom. Robot. ICCAR 2017. 740-744 (2017).

5. The European Parliament and the Council of the European Union: Machine Directive 2006/42/EC. (2006)

6. Finkemeyer, B.: Towards safe human-robot collaboration. 2017 22nd Int. Conf. Methods Model. Autom. Robot. MMAR 2017. 883-888 (2017).

7. Murashov, V., Hearl, F., Howard, J.: Working safely with robot workers: Recommendations for the new workplace. J. Occup. Environ. Hyg. 13, D61-D71 (2016).

8. Navarro, B., Cherubini, A., Fonte, A., Passama, R., Poisson, G., Fraisse, P.: An ISO10218-compliant adaptive damping controller for safe physical human-robot interaction. Proc. - IEEE Int. Conf. Robot. Autom. 2016-June, 3043-3048 (2016).

9. Rojas, R.A., Garcia, M.A.R., Wehrle, E., Vidoni, R.: A Variational Approach to Minimum-Jerk Trajectories for Psychological Safety in Collaborative Assembly Stations. IEEE Robot. Autom. Lett. 4, 823-829 (2019).

10. Vysocky, A., Wada, H., Kinugawa, J., Kosuge, K.: Motion planning analysis according to ISO/TS 15066 in human-robot collaboration environment. IEEE/ASME Int. Conf. Adv. Intell. Mechatronics, AIM. 2019-July, 151-156 (2019).

11. Lasota, P., Shah, J.: Analyzing the Effects of Human-Aware Motion Planning on CloseProximity Human-Robot Collaboration. Hum. Factors J. Hum. Factors Ergon. Soc. 57, 21-33 (2015).

12. Zanchettin, A.M., Ceriani, N.M., Rocco, P., Ding, H., Matthias, B.: Safety in HumanRobot Collaborative Manufacturing Environments: Metrics and Control. IEEE Trans. Autom. Sci. Eng. 13, 882-893 (2016).

13. Vicentini, F., Askarpour, M., Rossi, M.G., Mandrioli, D.: Safety Assessment of Collaborative Robotics Through Automated Formal Verification. IEEE Trans. Robot. 1-20 (2019).

14. Poot, L., Johansen, K., Gopinath, V.: Supporting risk assessment of human-robot collaborative production layouts: A proposed design automation framework. Procedia Manuf. 25, 543-548 (2018). 